\title{
Hybrid Specific Combining Ability in Relation to Parental General Combining Ability in Dolichos Bean (Lablab purpureus L.)
}

\author{
C.M. Keerthi ${ }^{1 *}$, S. Ramesh ${ }^{1}$, M. Byregowda ${ }^{2}$ and A. Mohan Rao ${ }^{1}$ \\ ${ }^{1}$ Department of Genetics and Plant Breeding, University of Agricultural Sciences (UAS), \\ GKVK, Bangalore-560 065, Karnataka, India \\ ${ }^{2}$ All India Coordinated Research Project on Pigeonpea, ZARS, UAS, Bangalore-560 065, \\ Karnataka, India \\ *Corresponding author
}

\begin{abstract}
Keywords
Combining ability, Dolichos bean,

Gene action, per se performance

Article Info

Accepted:

12 December 2017

Available Online:

10 January 2018 component traits, besides pinpointing the promising parents to be used in the creation of genetic variability for eventual use in development of suitable varieties in self-pollinated crops. Hence, a study was undertaken to assess combining ability of crosses and their relationship with their parental general combining ability and elicit mode of action of genes controlling pod yield and its component traits in Dolichos bean using 12 lines and 4 testers. The 48 crosses (developed through Line $\times$ Tester combination) and their parents were evaluated for seed yield and its component traits during 2013 and 2014 kharif seasons at experimental plots of University of Agricultural Sciences, Bengaluru. Significant differences were found among the lines for all the traits and among testers for all the characters except for racemes plant ${ }^{-1}$, justifying the selection of parents. Further, greater magnitude of $s c a$ variance for most of the traits studied in both years indicates greater importance of non-additive gene action for the inheritance of these traits. The lines RIL 185, HA 10-8 and tester RIL 60 were considered as best general combiners for most of the traits studied irrespective of the years. While, the crosses involving the line RIL 185 followed by RIL 332 and tester RIL 60 exhibited desirable sca effects in both years for most of the traits.
\end{abstract}

\section{A B S T R A C T}

The choice of parents in a breeding programme for hybridization is one of the most critical considerations, since the selection on the basis of performance does not provide clear information. Combining ability, play a significant role in crop improvement, since they help in characterizing the nature and magnitude of genetic effects governing yield and

\section{Introduction}

Dolichos bean (Lablab purpureus L. Sweet) commonly known as field bean, hyacinth bean, Indian bean, sem, butter bean, Egyptian kidney bean and lubia bean etc., belongs to the family Fabaceae and is one of the most ancient crops among the cultivated plants. The crop is documented by archaeo-botanical finds in India from 2000 to $1700 \mathrm{BC}$ at Hallur, the earliest Iron Age site in Karnataka, to 1200$300 \mathrm{BC}$ at the Veerapuram excavation site in Andhra Pradesh (Fuller, 2003). In India, Dolichos bean is primarily cultivated in 
Karnataka and adjoining districts of Tamil Nadu, Andhra Pradesh and Maharashtra (Mahadevu and Byre Gowda, 2005) for use as vegetable (immature pods and immature grains) and forage (NRC, 2006). It is cultivated either as a pure crop or intercropped with finger millet, groundnut, castor, corn, pearl millet or sorghum. Despite its importance as a multi-purpose crop and ability to withstand drought better than common bean and cowpea (Maass et al., 2010), and adapt to acidic (Mugwira and Haque, 1993) and saline soils (Murphy and Colucci, 1999), Dolichos bean truly qualifies as 'underutilized' crop as evidenced by limited efforts for its genetic improvement.

Pure-limes are the only cultivar option in Dolichos bean as it is predominantly selffertilizing species (Shivashankar and Kulkarni, 1989). Pedigree breeding is the most preferred method of developing pure-line cultivars in Dolichos bean. Pedigree breeding is a method of combining several desirable traits in a single genotype (pure-line) through selection from segregating generations derived from two/three/four/multi parent crosses involving parents harbouring desired traits.

Very often plant breeders are controlled with the task of selecting suitable parents for effecting crosses and choosing only a few highly productive crosses from a large number of crosses to maximise the probability of isolating desirable pure-lines. Combining ability (CA) is one of the widely accepted criteria for choosing the parents for effecting crosses. Apart from providing an objective criterion for choosing parents, CA also provides useful clues about mode of action of genes controlling economically important traits. The greatest advantage of CA approach for genetic analysis is that it is statistically robust and genetically neutral (Arunachalam, 1981). Precise knowledge on the nature of genetic control of target traits in working germplasm is a pre-requisite for designing suitable selection strategy.

Under these premises, the present investigation was carried out to assess combining ability of crosses and their relationship with their parental general combining ability and elicit mode of action of genes controlling pod yield and its component traits in Dolichos bean.

\section{Materials and Methods}

\section{Plant material and experimental design}

The base material for the study consisted of four inbred lines such as RIL 21, RIL 25, RIL 60 and RIL 180 designated as testers; 12 phenotypically diverse inbred lines which include two released varieties (HA 3 and HA 4), six advanced breeding lines (HA 11-3, HA 10-8, FPB 3, FPB 8, FPB 15 and FPB 21) and four recombinant inbred lines (RIL 11, RIL 162, RIL 185 and RIL 332) designated as lines (Table 1). The 12 inbred lines were crossed to four testers during 2012 rainy season. The 48 $\mathrm{F}_{1}$ 's and their parents (12 lines and 4 testers) along with two check varieties such as HA 3 and HA 4 were evaluated in a randomised block design with two replications during 2013 and 2014 rainy seasons at the experimental plots of Department of Genetics and Plant Breeding, University of Agricultural Sciences (UAS), Bengaluru, India.

The experimental plotsare located at $12^{\circ} 58^{\prime}$ latitude North, $77^{\circ} 35^{\prime}$ longitude East and an altitude of 930 meters above sea level. The annual rainfall ranges from $679.1 \mathrm{~mm}$ to 888.9 $\mathrm{mm}$. The data on average monthly minimum and maximum temperature, bright sunshine hrs and relative humidity are furnished in Table 2. The $48 \mathrm{~F}_{1}$ 'sand 12 inbred lines were planted in a single row of $3 \mathrm{~m}$ length with a spacing of $0.6 \mathrm{~m}$ between rows and $0.3 \mathrm{~m}$ between plants within a row. A total of 12 
plants were maintained in each row. Recommended production practices were followed for raising a good crop.

\section{Collection of data}

The data were collected on five randomly selected plants (avoiding border plants) in each of the $48 \mathrm{~F}_{1}$ 's, parents and check varieties and in each replication on days to flowering, racemes plant ${ }^{-1}$, pods plant ${ }^{-1}$, dry pod weight plant $^{-1}$, dry seed weight plant $^{-1}$ and fresh pod weight plant $^{-1}$.

\section{Statistical analysis}

The mean of two replications were used for thecombining ability analyses [9] Kempthorne (1957). Analysis of variance for CA, CA effects and components of genotypic variance were computed by using computer software program Windowstat 8.0 (developed by Indostat services 18.0, Ameerpet, Hyderabad, India).

\section{Results and Discussion}

\section{Pooled analysis of variance}

From the pooled analysis of variance (Table 1), indicated significant differences among the lines for all the traits and among testers for all the characters except for racemes plant $^{-1}$, justifying the selection of parents for CA analysis. Dethe et al., (2008) in mungbean, Iqbal et al., (2010) in common bean and Das et al., (2014) in Dolichos bean reported significant difference among parents and crosses for days to flowering, racemes plant ${ }^{-1}$, pods plant ${ }^{-1}$, pod and seed yield plant $^{-1}$. Significant mean sum of squares due to genotypes $\times$ years $\quad(\mathrm{P}<0.01) \quad$ indicated differential performance of parents and crosses across twoyears. On the other hand, non-significant mean sum of squares due parents $\times$ years interaction for most of the traits suggested comparable per se performance of parents. However, crosses interacted significantly over the years for most of the traits.

Significant mean sum of squares due to line effect (for days to flowering, dry seed weight and fresh pod weight plant $^{-1}$ ), tester effect and line $\times$ tester $(\mathrm{L} \times \mathrm{T})$ effect for all the traits suggested importance of both general and specific combining ability for these traits. The significance of the interaction arising from line effect with season for days to flowering and $\mathrm{L} \times \mathrm{T}$ effect with the years for all the traits except for days to flowering and fresh pod weight plant $^{-1}$ revealed that the alleles controlling the gca and sca behaved differently in the different years. The presence of significant line effect, tester effect or $\mathrm{L} \times \mathrm{T}$ effect $x$ years interaction have been reported by Matzinger et al., (1959) in maize, Kunkaew et al., (2006) in azukibean and Iqbal et al., (2010) in rajmesh for seed weight plant ${ }^{-1}$, with contradictory reports revealing non-significant interaction of line effect, tester effect or $\mathrm{L} \times \mathrm{T}$ effect $\times$ years for pods plant ${ }^{-1}$. Comparison of relative magnitude of $\mathrm{gca}$ and $\mathrm{sca}$ variances indicated greater magnitude of $s c a$ variance for most of the traits studied in both years, thereby indicating greater importance of nonadditive gene action for the inheritance of these traits.

Importance of non-additive gene action forpod and seed weight plant ${ }^{-1}$ have been reported by Sofi et al., (2006) field pea, Barelli et al., (2000) in common bean, Saleem (2009) in faba bean and Iqbal et al., (2010) in rajmesh and for racemes plant ${ }^{-1}$ have been reported by Singh and Singh (1981), Singh et al.,(1980, 1986) in lablab bean. However, the average degree of dominance was in the range of partial to over-dominance for all the traits studied (Table 2), which was revealed by Sofi et al., (2006) in field pea and Iqbal et al., (2010) in rajmesh. 
Practical utility of CA lies in the prediction of performance of yet untested $\mathrm{F}_{1}$ hybrids. Prediction power of gca is fairly high provided sca is absent or of very low magnitude. When both gca and sca are important gca serves the purpose of only short-listing parents further testing in specific combination. An important principle repeatedly overlooked frequently, is that estimates of gca and sca are relative to and dependent on the particular set of inbred lines included in the experiment.

Sprague and Tatum (1985) interpreted CA in terms of mode of action of genes. The differences in gca of lines are due to $\sigma_{\text {A }}^{2}$ and $\sigma^{2}$ AA. The differences in sca of crosses assemble due to non-additive $\sigma_{\mathrm{g}}^{2}$. In selected set of maternal, gca is less important than that of sca, whereas reverse is true for unselected maternal (Alam and Newaz, 2005).

\section{General combining ability (gca) effects}

The parental combining ability of parents (Table 3 ) revealed that among lines, HA 3 and FPB 21 and among testers, RIL 25 were good general combiners for early flowering in both years. The line, FPB 15 and RIL 332 exhibiting good combining ability for racemes plant $^{-1}$ in 2013 rainy season only.

The lines RIL 185 and HA 10-8 were good general combiners as it exhibited significant positive $g c a$ effects for racemes plant ${ }^{-1}$ (in 2014 rainy season), pods plant ${ }^{-1}$, dry pod and seed weight plant ${ }^{-1}$ and fresh pod weight plant $^{-1}$ in both years. However, line HA 10-8 showed itself as a good general combiner for dry pod and seed weight plant ${ }^{-1}$ in 2014 rainy season only.

Among testers, RIL 60 showed good general combiner for racemes plant $^{-1}$ (in 2014 rainy season only), pods plant ${ }^{-1}$, fresh pod weight plant $^{-1}$, dry pod and seed weight plant ${ }^{-1}$ in both years.
Though none of the parents showed good general combining ability for all the traits, the lines RIL 185, HA 10-8 and tester RIL 60 were considered as best general combiners for most of the traits studied irrespective of the years.

Different types of combining ability in different years were also found for most of the genotypes. These differential results may be due to genotype-environment interaction. The similar results of differential types of combining ability in different sowing dates were also noticed by Alam and Newaz (2005) in lablab bean.

\section{Specific combining ability (sca) effects}

The estimates of $s c a$ effect revealed the importance of gene interaction for the characters studied. The cross combination RIL $332 \times$ RIL 180 in 2013 rainy season and cross combination involved RIL 60 as a tester parent with lines RIL 11, FPB 21 and RIL 332 in 2014 rainy season had the highest significant $s c a$ effect in desirable direction for early flowering. The crosses HA $10-8 \times$ RIL 60, RIL $332 \times$ RIL 180 and FPB $3 \times$ RIL 60 showed significant positive sca effect for pods plant $^{-1}$, fresh pod weight plant ${ }^{-1}$, dry pod and seed weight plant $^{-1}$ in 2013 rainy season. On the other hand, crosses such as RIL $185 \times$ RIL 25 and RIL $162 \times$ RIL 60 exhibited high sca effect for pods plant $^{-1}$, fresh pod weight plant $^{-1}$, dry pod and seed weight plant $^{-1}$ in 2014 rainy season.

However, some other crosses gave significant positive sca effects in 2013 rainy season but non-significant or significant negative sca effects in 2014 rainy season for same traits and vice versa (Table 4). Alam and Newaz (2005) in lablab bean and Sofi et al., (2006) in field pea also reported the crosses having differential response of $s c a$ effects in different environments/sowing dates. 
Table.1 Pooled analysis of variance for quantitative traits in Dolichos bean

\begin{tabular}{|c|c|c|c|c|c|c|c|}
\hline Source of variation & df & $\begin{array}{l}\text { Days to } \\
\text { flowering }\end{array}$ & $\begin{array}{c}\text { Racemes } \\
\text { plant }^{-1}\end{array}$ & Pods plant $^{-1}$ & $\begin{array}{l}\text { Dry pod weight } \\
\text { plant }^{-1}\end{array}$ & $\begin{array}{c}\text { Dry seed weight } \\
\text { plant }^{-1}\end{array}$ & $\begin{array}{c}\text { Fresh pod weight } \\
\text { plant }^{-1}\end{array}$ \\
\hline Replications & 1 & 10.041 & 0.42 & 149.71 & 0.68 & 98.68 & 46.25 \\
\hline Years & 1 & $500.08 * *$ & 0.44 & $3568.05^{* *}$ & $4484.65 * *$ & $218.93 *$ & $5673.95 * *$ \\
\hline Replication $\times$ Years & 1 & $58.43 *$ & 3.22 & 176.51 & 64.62 & $320.45 * *$ & $310.88^{*}$ \\
\hline Genotypes & 63 & $154.47 * *$ & $16.49 * *$ & $1572.26^{* *}$ & $1466.57 * *$ & $558.39 * *$ & $2225.49 * *$ \\
\hline Parents (P) & 15 & $99.62 * *$ & $4.83 * *$ & $600.19 * *$ & $1271.11^{* *}$ & $553.93 * *$ & $2783.70 * *$ \\
\hline Lines $(\mathrm{L})$ & 11 & $86.85^{* *}$ & $4.73 * *$ & $289.01 * *$ & $795.51 * *$ & $361.91 * *$ & $1519.39 * *$ \\
\hline Testers (T) & 3 & $175.91 * *$ & 2.41 & $1356.05 * *$ & $2418.98 * *$ & $1036.35^{* *}$ & $5471.46^{* *}$ \\
\hline Lvs T & 1 & 11.21 & $13.20 * *$ & $1755.71 * *$ & $3059.13 * *$ & $1218.92 * *$ & $8627.86^{* *}$ \\
\hline P vs C & 1 & $401.65 * *$ & $196.21 * *$ & $13686.05 * *$ & $19577.64 * *$ & $8123.05 * *$ & $26600.32 * *$ \\
\hline Crosses (C) & 47 & $166.72 * *$ & $16.38 * *$ & $1624.76^{* *}$ & $1143.61 * *$ & $398.87 * *$ & $1528.72 * *$ \\
\hline Line effect & 11 & $415.85^{* *}$ & 15.80 & 1181.71 & 1066.03 & $471.00 *$ & $1805.05^{*}$ \\
\hline Tester effect & 3 & $267.28 *$ & $48.40 *$ & $10722.46 * *$ & $6558.73^{* *}$ & $2150.36^{* *}$ & $7909.15^{* *}$ \\
\hline Line $\times$ Tester effect & 33 & $74.53 * *$ & $13.67 * *$ & $945.37 * *$ & $677.18 * *$ & $215.61 * *$ & $856.57 * *$ \\
\hline Years $\times$ Genotypes & 63 & $30.27 * *$ & $8.15^{* *}$ & $149.06^{* *}$ & $217.99 * *$ & $89.31 * *$ & $307.92 * *$ \\
\hline Years $\times$ Parents & 15 & $35.91 * *$ & 0.74 & 48.40 & 142.39 & 43.38 & 75.61 \\
\hline Years $\times$ Lines & 11 & $41.54 * *$ & 0.83 & 50.84 & 131.54 & 44.16 & 93.12 \\
\hline Years $\times$ Testers & 3 & 10.57 & 0.27 & 21.84 & 33.87 & 4.53 & 1.36 \\
\hline Years $\times$ Lvs T & 1 & 50.02 & 1.18 & 101.21 & $587.26^{*}$ & 151.32 & $105.72 *$ \\
\hline Years $\times$ Pvs C & 1 & 21.00 & 0.01 & 7.53 & $1040.86^{* * *}$ & $436.72 * *$ & $352.91 * *$ \\
\hline Years $\times$ Crosses & 47 & $28.67 * *$ & $10.69^{* *}$ & $184.20 * *$ & $224.61 * *$ & $96.56^{* *}$ & 381.11 \\
\hline Years $\times$ Line effect & 11 & $47.90^{*}$ & 6.28 & 152.71 & 181.10 & 50.65 & 245.54 \\
\hline Years $\times$ Tester effect & 3 & 39.01 & 18.53 & 315.36 & 284.33 & 182.06 & 273.62 \\
\hline Years $\times(L \times T)$ effect & 33 & 21.31 & $11.45^{* *}$ & $182.78 * *$ & $233.68 * *$ & $104.09 * *$ & $436.07 * *$ \\
\hline Pooled error & 126 & 13.98 & 1.44 & 51.75 & 111.47 & 45.22 & 74.64 \\
\hline
\end{tabular}

Table.2 Estimates of combining ability effects, components of variance and narrow-sense heritability $\left(\mathrm{h}^{2}-\mathrm{ns}\right)$ for quantitative traits in Dolichos bean

\begin{tabular}{|c|c|c|c|c|c|c|c|c|c|c|c|c|}
\hline \multirow[t]{2}{*}{ Parameters } & \multicolumn{2}{|c|}{ Days to flowering } & \multicolumn{2}{|c|}{ Racemes plant $^{-1}$} & \multicolumn{2}{|c|}{ Pods plant $^{-1}$} & \multicolumn{2}{|c|}{$\begin{array}{l}\text { Dry pod weight } \\
\text { plant }^{-1}\end{array}$} & \multicolumn{2}{|c|}{$\begin{array}{l}\text { Dry seed weight } \\
\text { plant }^{-1}\end{array}$} & \multicolumn{2}{|c|}{$\begin{array}{c}\text { Fresh pod weight } \\
\text { plant }^{-1}\end{array}$} \\
\hline & 2013 & 2014 & 2013 & 2014 & 2013 & 2014 & 2013 & 2014 & 2013 & 2014 & 2013 & 2014 \\
\hline$\sigma^{2}$ GCA & 10.73 & 11.59 & 0.97 & 1.63 & 188.09 & 192.07 & 162.07 & 76.82 & 56.78 & 26.76 & 119.37 & 191.10 \\
\hline$\sigma^{2} \mathrm{SCA}$ & 8.96 & 24.99 & 7.29 & 3.83 & 239.20 & 273.13 & 147.80 & 196.16 & 45.92 & 68.70 & 137.42 & 434.26 \\
\hline GCA / SCA & 1.20 & 0.46 & 0.13 & 0.43 & 0.79 & 0.70 & 1.10 & 0.39 & 1.24 & 0.39 & 0.87 & 0.44 \\
\hline$\sigma_{A}^{2}$ & 21.46 & 23.17 & 1.94 & 3.26 & 376.18 & 384.15 & 324.13 & 153.64 & 113.56 & 53.52 & 238.73 & 382.20 \\
\hline$\sigma_{D}^{2}$ & 8.96 & 24.99 & 7.29 & 3.83 & 239.20 & 273.13 & 147.80 & 196.16 & 45.92 & 68.70 & 137.42 & 434.26 \\
\hline$\sigma_{\mathrm{E}}^{2}$ & 11.42 & 2.56 & 0.69 & 0.75 & 22.92 & 28.83 & 53.40 & 58.06 & 21.89 & 23.34 & 39.41 & 35.23 \\
\hline$\sigma_{P}^{2}$ & 41.84 & 50.72 & 9.93 & 7.83 & 638.30 & 686.11 & 525.34 & 407.86 & 181.36 & 145.56 & 415.56 & 851.68 \\
\hline$\sqrt{ } \sigma_{\mathrm{D} /}^{2} \sigma_{\mathrm{A}}^{2}$ & 0.65 & 1.04 & 1.94 & 1.08 & 0.80 & 0.84 & 0.68 & 1.13 & 0.64 & 1.13 & 0.76 & 1.06 \\
\hline$h^{2}-n s(\%)$ & 51.29 & 45.69 & 19.57 & 41.64 & 58.94 & 55.99 & 61.70 & 37.67 & 62.61 & 36.77 & 57.45 & 44.87 \\
\hline
\end{tabular}


Table.3 Estimates of general combining ability effects of lines and testers for quantitative traits in Dolichos bean

\begin{tabular}{|c|c|c|c|c|c|c|c|c|c|c|c|c|}
\hline \multirow[t]{2}{*}{ Genotypes } & \multicolumn{2}{|c|}{ Days to flowering } & \multicolumn{2}{|c|}{ Racemes plant $^{-1}$} & \multicolumn{2}{|c|}{ Pods plant $^{-1}$} & \multicolumn{2}{|c|}{ Dry pod weight plant ${ }^{-1}$} & \multicolumn{2}{|c|}{${ }^{\text {Dry seed weight plant }}$} & \multicolumn{2}{|c|}{ Fresh pod weight plant } \\
\hline & 2013 & 2014 & 2013 & 2014 & 2013 & 2014 & 2013 & 2014 & 2013 & 2014 & 2013 & 2014 \\
\hline \multicolumn{13}{|l|}{ Lines } \\
\hline HA 3 & $-7.50 * *$ & $-3.41 * *$ & $-1.08^{*}$ & $-1.76^{* *}$ & $-8.51 * *$ & -4.96 & -6.32 & -1.67 & -4.22 & -1.51 & 3.47 & $-6.91^{*}$ \\
\hline HA 4 & $-6.05 * *$ & $-2.66^{* *}$ & -0.46 & -0.41 & 0.84 & $-6.23 *$ & -5.32 & -3.42 & $-4.72 *$ & -4.43 & -6.28 & -2.00 \\
\hline HA 11-3 & $-5.55 * *$ & $-3.01 * *$ & -0.04 & -0.63 & -3.41 & 5.28 & -2.32 & -0.03 & -2.05 & -0.31 & 4.80 & $8.54 * *$ \\
\hline RIL 11 & -0.70 & $2.44 * *$ & $-1.04 *$ & $-0.87 *$ & $-15.18 * *$ & $-8.67 * *$ & -5.02 & -3.22 & -1.48 & -0.37 & -4.53 & $-12.87 * *$ \\
\hline RIL 185 & $13.35 * *$ & $11.89 * *$ & 0.42 & $2.99 * *$ & $19.32 * *$ & $7.78 * *$ & $27.76^{* *}$ & $12.83 * *$ & $16.64 * *$ & $9.97 * *$ & $21.00 * *$ & $13.06 * *$ \\
\hline FPB 8 & -1.82 & -0.24 & $-1.29 * *$ & -0.56 & $-9.16^{* *}$ & $-6.43^{*}$ & -6.98 & $-8.80 *$ & $-5.28 *$ & $-6.01 *$ & $-14.14 * *$ & $-11.08 * *$ \\
\hline FPB 15 & -0.70 & -1.14 & $1.67 * *$ & 0.79 & -2.81 & -4.41 & 0.83 & -1.63 & 1.85 & -0.20 & $-9.62 * *$ & $-9.63 * *$ \\
\hline RIL 332 & $5.00^{* *}$ & $-2.19 * *$ & $1.69 * *$ & -0.09 & 2.27 & 2.21 & -5.92 & -5.97 & -4.53 & -1.95 & -2.88 & $-9.54 * *$ \\
\hline FPB 3 & $7.55^{* *}$ & $2.51 * *$ & -0.69 & $1.07 *$ & -1.41 & -2.61 & $11.63 * *$ & 1.22 & $8.16^{* *}$ & 1.63 & $6.32 *$ & 0.00 \\
\hline RIL 162 & 2.23 & 0.51 & -0.77 & $-1.12 *$ & 15.47 ** & $8.67 * *$ & -5.63 & -0.41 & -3.35 & -3.23 & $-13.64 * *$ & -2.92 \\
\hline HA 10-8 & 0.20 & -0.19 & 0.67 & $1.06^{*}$ & $9.17 * *$ & $16.14^{* *}$ & 5.73 & $13.35^{* *}$ & 4.04 & $6.90 * *$ & $14.15^{* *}$ & $27.21 * *$ \\
\hline FPB 21 & $-6.02 * *$ & $-4.51 * *$ & $0.92 *$ & -0.49 & $-6.58 * *$ & $-6.77 *$ & $-8.46^{*}$ & -2.25 & $-5.06^{*}$ & -0.49 & 1.34 & $6.16^{*}$ \\
\hline C.D@ $95 \%$ & 3.40 & 1.61 & 0.84 & 0.87 & 4.82 & 5.40 & 7.35 & 7.66 & 4.71 & 4.86 & 6.31 & 5.97 \\
\hline C.D@ $99 \%$ & 4.54 & 2.15 & 1.12 & 1.16 & 6.43 & 7.21 & 9.81 & 10.23 & 6.28 & 6.48 & 8.43 & 7.97 \\
\hline \multicolumn{13}{|l|}{ Testers } \\
\hline RIL 21 & -0.03 & $-2.40 * *$ & 0.48 & -0.26 & $-4.91 * *$ & $-10.72 * *$ & $-6.29 * *$ & $-7.13 * *$ & $-3.71 * *$ & $-5.66 * *$ & $-5.11 * *$ & $-5.65^{* *}$ \\
\hline RIL 25 & -1.84 & $-1.80^{* *}$ & $-1.53 * *$ & $-0.81 * *$ & $-8.88 * *$ & $-10.02 * *$ & $-9.04 * *$ & $-6.69 * *$ & $-4.02 * *$ & -2.41 & $-7.76^{* *}$ & $-14.31 * *$ \\
\hline RIL 60 & $2.43^{*}$ & $4.43 * *$ & $0.59 *$ & $1.94 * *$ & $22.07 * *$ & $22.42 * *$ & $20.41 * *$ & $14.01 * * *$ & $12.03 * *$ & $7.78^{* *}$ & $16.75^{* *}$ & $20.10 * *$ \\
\hline RIL 180 & -0.56 & -0.22 & 0.45 & $-0.87 * *$ & $-8.28 * *$ & -1.68 & $-5.076^{*}$ & -0.19 & $-4.30 * *$ & 0.30 & $-3.88^{*}$ & -0.13 \\
\hline C.D@ 95\% & 1.96 & 0.93 & 0.48 & 0.50 & 2.78 & 3.12 & 4.24 & 4.43 & 2.72 & 2.81 & 3.64 & 3.44 \\
\hline C.D@ 99\% & 2.62 & 1.24 & 0.65 & 0.67 & 3.71 & 4.16 & 5.66 & 5.91 & 3.63 & 3.74 & 4.86 & 4.60 \\
\hline
\end{tabular}

$*$ Significant at $\mathrm{P}=0.05 \quad * *$ Significant at $\mathrm{P}=0.01$

Table.4 Top ranking specific cross combinations on the basis of sca effects, their per se performance and their parental gca status

\begin{tabular}{|c|c|c|c|c|c|c|c|c|}
\hline \multirow[t]{2}{*}{ Traits } & \multicolumn{4}{|c|}{2013} & \multicolumn{4}{|c|}{2014} \\
\hline & Crosses & sca effect & Per se value & gea status & Crosses & sca effect & Per se value & gea status \\
\hline \multirow{5}{*}{$\begin{array}{l}\text { Days to } \\
\text { flowering }\end{array}$} & RIL $332 \times$ RIL 180 & $-8.07 *$ & 48.40 & $\mathrm{H} \times \mathrm{L}$ & RIL $11 \times$ RIL 60 & $-8.25 * *$ & 53.10 & $\mathrm{H} \times \mathrm{L}$ \\
\hline & RIL $185 \times$ RIL 21 & -6.24 & 59.10 & $\mathrm{~L} \times \mathrm{H}$ & FPB $21 \times$ RIL 60 & $-6.60 * *$ & 47.80 & $\mathrm{~L} \times \mathrm{L}$ \\
\hline & RIL $162 \times$ RIL 60 & -5.78 & 50.90 & $\mathrm{H} \times \mathrm{L}$ & RIL $332 \times$ RIL 60 & $-5.23 * *$ & 51.50 & $\mathrm{H} \times \mathrm{L}$ \\
\hline & RIL $11 \times$ RIL 60 & -5.65 & 48.10 & $\mathrm{H} \times \mathrm{L}$ & RIL $185 \times$ RIL 21 & $-5.07 * *$ & 58.90 & $\mathrm{~L} \times \mathrm{H}$ \\
\hline & FPB $3 \times$ RIL 180 & -4.32 & 54.70 & $\mathrm{~L} \times \mathrm{L}$ & FPB $3 \times$ RIL 180 & $-4.78 * *$ & 52.00 & $\mathrm{~L} \times \mathrm{L}$ \\
\hline \multirow{5}{*}{$\begin{array}{l}\text { Racemes } \\
\text { plant }^{-1}\end{array}$} & HA $11-3 \times$ RIL 180 & $8.75 * *$ & 17.42 & $\mathrm{~L} \times \mathrm{L}$ & RIL $162 \times$ RIL 60 & $3.95^{* *}$ & 13.10 & $\mathrm{H} \times \mathrm{L}$ \\
\hline & HA $3 \times$ RIL 60 & $4.57 * *$ & 12.33 & $\mathrm{H} \times \mathrm{L}$ & HA $10-8 \times$ RIL 60 & $3.46^{* *}$ & 14.80 & $\mathrm{~L} \times \mathrm{L}$ \\
\hline & RIL $11 \times$ RIL 180 & $2.84 * *$ & 10.50 & $\mathrm{H} \times \mathrm{L}$ & RIL $11 \times$ RIL 21 & $3.40^{* *}$ & 10.60 & $\mathrm{H} \times \mathrm{H}$ \\
\hline & RIL $185 \times$ RIL 21 & $2.60^{* * *}$ & 11.75 & $\mathrm{~L} \times \mathrm{H}$ & HA $11-3 \times$ RIL 25 & $3.36^{* *}$ & 10.25 & $\mathrm{~L} \times \mathrm{H}$ \\
\hline & FPB $3 \times$ RIL 21 & $2.29 * *$ & 10.33 & $\mathrm{~L} \times \mathrm{H}$ & FPB $15 \times$ RIL 60 & $3.03 * *$ & 14.10 & $\mathrm{~L} \times \mathrm{L}$ \\
\hline \multirow[t]{5}{*}{ Pods plant $^{-1}$} & HA $10-8 \times$ RIL 60 & $33.60^{* *}$ & 111.80 & $\mathrm{~L} \times \mathrm{L}$ & RIL $162 \times$ RIL 60 & $41.16^{* *}$ & 126.88 & $\mathrm{H} \times \mathrm{L}$ \\
\hline & HA $4 \times$ RIL 21 & $25.31 * *$ & 68.20 & $\mathrm{H} \times \mathrm{H}$ & RIL $185 \times$ RIL 25 & $32.62^{* * *}$ & 85.00 & $\mathrm{~L} \times \mathrm{H}$ \\
\hline & RIL $185 \times$ RIL 21 & -6.27 & 55.10 & $\mathrm{~L} \times \mathrm{H}$ & HA $10-8 \times$ RIL 60 & $23.89^{* *}$ & 117.07 & $\mathrm{~L} \times \mathrm{L}$ \\
\hline & RIL $332 \times$ RIL 180 & $20.16^{* *}$ & 61.10 & $\mathrm{H} \times \mathrm{L}$ & FPB $3 \times$ RIL 60 & $21.51^{* *}$ & 95.93 & $\mathrm{~L} \times \mathrm{L}$ \\
\hline & FPB $3 \times$ RIL 60 & $19.97 * *$ & 87.60 & $\mathrm{~L} \times \mathrm{L}$ & RIL $332 \times$ RIL 21 & $20.19 * *$ & 66.30 & $\mathrm{H} \times \mathrm{H}$ \\
\hline \multirow{5}{*}{$\begin{array}{l}\text { Dry pod } \\
\text { weight plant }^{-1}\end{array}$} & HA $10-8 \times$ RIL 60 & $37.02 * *$ & 124.10 & $\mathrm{~L} \times \mathrm{L}$ & RIL $185 \times$ RIL 25 & 44.29 ** & 117.42 & $\mathrm{~L} \times \mathrm{H}$ \\
\hline & RIL $185 \times$ RIL 25 & $19.44 *$ & 99.10 & $\mathrm{~L} \times \mathrm{H}$ & RIL $162 \times$ RIL 60 & $25.59^{* *}$ & 106.17 & $\mathrm{H} \times \mathrm{L}$ \\
\hline & RIL $332 \times$ RIL 180 & $17.55^{*}$ & 67.50 & $\mathrm{H} \times \mathrm{L}$ & FPB $8 \times$ RIL 180 & $19.09^{*}$ & 77.08 & $\mathrm{H} \times \mathrm{L}$ \\
\hline & FPB $21 \times$ RIL 180 & $17.49 *$ & 64.90 & $\mathrm{~L} \times \mathrm{L}$ & FPB $21 \times$ RIL 21 & $16.76^{*}$ & 74.38 & $\mathrm{~L} \times \mathrm{H}$ \\
\hline & FPB $3 \times$ RIL 60 & $17.42 *$ & 110.40 & $\mathrm{~L} \times \mathrm{L}$ & FPB $15 \times$ RIL 25 & $16.25^{*}$ & 74.92 & $\mathrm{~L} \times \mathrm{H}$ \\
\hline \multirow{5}{*}{$\begin{array}{l}\text { Dry seed } \\
\text { weight plant }^{-1}\end{array}$} & HA $10-8 \times$ RIL 60 & $22.25^{* *}$ & 78.74 & $\mathrm{~L} \times \mathrm{L}$ & RIL $185 \times$ RIL 25 & $31.10^{* * *}$ & 79.42 & $\mathrm{~L} \times \mathrm{H}$ \\
\hline & FPB $3 \times$ RIL 60 & $14.83 * *$ & 75.44 & $\mathrm{~L} \times \mathrm{L}$ & RIL $162 \times$ RIL 60 & $13.10^{* * *}$ & 58.42 & $\mathrm{H} \times \mathrm{L}$ \\
\hline & FPB $21 \times$ RIL 180 & $12.17^{*}$ & 43.23 & $\mathrm{~L} \times \mathrm{L}$ & RIL $11 \times$ RIL 180 & $9.81 *$ & 50.50 & $\mathrm{H} \times \mathrm{L}$ \\
\hline & RIL $185 \times$ RIL 25 & $11.00^{*}$ & 64.04 & $\mathrm{~L} \times \mathrm{H}$ & FPB $15 \times$ RIL 25 & 9.69 & 47.83 & $\mathrm{~L} \times \mathrm{H}$ \\
\hline & RIL $332 \times$ RIL 180 & 6.97 & 38.56 & $\mathrm{H} \times \mathrm{L}$ & FPB $8 \times$ RIL 180 & 9.20 & 44.25 & $\mathrm{H} \times \mathrm{L}$ \\
\hline \multirow{5}{*}{$\begin{array}{l}\text { Fresh pod } \\
\text { weight plant }^{-1}\end{array}$} & HA $10-8 \times$ RIL 60 & $27.85^{* *}$ & 146.82 & $\mathrm{~L} \times \mathrm{L}$ & RIL $185 \times$ RIL 25 & $44.17^{* * *}$ & 141.75 & $\mathrm{~L} \times \mathrm{H}$ \\
\hline & RIL $332 \times$ RIL 180 & $21.46 * *$ & 102.77 & $\mathrm{H} \times \mathrm{L}$ & FPB $21 \times$ RIL 180 & $29.46 * *$ & 134.33 & $\mathrm{~L} \times \mathrm{L}$ \\
\hline & FPB $21 \times$ RIL 180 & $17.62 * *$ & 103.15 & $\mathrm{~L} \times \mathrm{L}$ & HA $4 \times$ RIL 180 & $25.63^{* * *}$ & 122.33 & $\mathrm{H} \times \mathrm{L}$ \\
\hline & FPB $3 \times$ RIL 60 & $16.01 *$ & 127.15 & $\mathrm{~L} \times \mathrm{L}$ & FPB $3 \times$ RIL 60 & $23.07 * *$ & 142.00 & $\mathrm{~L} \times \mathrm{L}$ \\
\hline & HA $4 \times$ RIL 21 & $13.70 *$ & 90.37 & $\mathrm{H} \times \mathrm{H}$ & FPB $15 \times$ RIL 25 & $23.60^{* * *}$ & 98.50 & $\mathrm{~L} \times \mathrm{H}$ \\
\hline
\end{tabular}


The best five cross combinations for yield and its attributing traits on the basis of sca revealed that line RIL 185 was involved in maximum number of cross combination (10) followed by RIL 332 and FPB 3 (6) and tester RIL 60 (22) followed by RIL 180 (17) revealing desirable $s c a$ effects in both years. While assessing the performance of parents on the basis of general combining ability, it was observed that most of the desirable cross combinations involved high $\times$ high, high $\times$ low/low $x$ high and low $\times$ low general combiners, which have also been reported by several workers in most of the crop species (Ram and Rajput (1999) in French bean; Ganesamurthy and Seshadri (2002) in soybean; Alam and Newaz (2005) in lablab bean; Iqbal et al., (2010) in rajmesh). These results suggested the involvement of both additive and non-additive gene actions in the expression of the characters of Dolichos bean.

These parents are expected to give heterotic combinations resulting from the non-additive gene action and also results in the evolution of transgressive segregants for high yielding genotypes ensuing from complementary epitasis, additive type of gene action and recombination of latent genetic variability hidden in the heterogenetic blocks of such crosses. Normally, sca alone would not contribute tangibly in the improvement of self-pollinated crops, except where commercial heterosis is feasible. However, sca resulting from the heterozygosity of polygenes governing yield and yield components may result in the evolution of recombinants possessing desirable gene aggregates in a homozygous line.

Early elimination of a less productive crosses helps in efficient utilization of resources and allows handling of a reasonably large segregating populations of only a few crosses for maximise isolated purelines. Hybridization between chosen parents is only the beginning of a long process of selection and isolation of desirable recombinant inbred lines.

Rawlings and Cockerham (1962) suggested that a variety having best gca should be used as a third parent for a triple cross hybrid. Such triple cross hybrids have an element of gamete selection, since the superior performance of triple cross $F_{1}$ reflects the combination of a superior gamete from the segregating single cross with the genotype of non-segregating variety used as third parent. Stadler (1939) argued that frequency of superior gametes is much higher $\left(\mathrm{q}^{2}\right)$ than the frequency of superior zygotes $(\mathrm{q})$. Therefore triple cross hybrids provide a more efficient mating system for obtaining lines with higher performance.

The dissimilarities between per se performance of the crosses and their sca effects suggested that both parameters such as per se performance and sca effects should be considered simultaneously in selecting suitable hybrids and their advanced generations. Genotypic expressions of the hybrids were also found to be different in different years indicating the presence of genotype-years interaction. Thus, plant breeders should also think about stable genotypes in different years before going on with selection of suitable advanced generation.

\section{References}

Alam, M. M., and Newaz, M. A. 2005. Combining ability for flower and pod characters of lablab bean under two sowing environments. Asian J. Plant Science. 4(6): 603-607.

Arunachalam, V., and Reddy, B. B. 1981. Evaluation of heterosis through combining ability in pearl millet. II. Multiple crosses. Indian Journal of 
Genetics. 41: 73-81.

Barelli, M. A. A., Vidigal, M. C. G., Amaral, A. T. J., Filho, P. S. V., and Scapim, C. A. 2000.Diallel analysis of the combining ability of common bean (Phaseolus vulgaris L.) cultivars. Brazilian Arch. Biol. Technology. 43: 409-414.

Das, I., Seth, T., Durwas, S.V., Dutta, S., Chattopadhyay, A., and Singh, B. 2014. Gene action and combining ability for yield and yield component traits in dolichos bean (Dolichos lablab var. typicus). Sabrao J. Breeding and Genetics. 46(2): 293-304.

Dethe, A. M., Patil, J. V., and Misal, A. M. 2008. Combining ability analysis inmungbean. J. Food Legumes. 21(3): 200-201.

Fuller, D. Q., 2003. African crops in prehistoric South Asia: A critical review In: (Eds.) K. Neumann., A. Butler and S. Kahlheber. Food, fuel, fields progress in African archaeobotany, Heinrich-Barth- Institute, Köln, Germany, Africa Praehistorica, 15, Pp. 239-271.

Ganesamurthy, K., and Seshadri, P. 2002. Diallel analysis in soybean (Glycine max L.). Madras Agriculture Journal. 89: 14-17.

Iqbal, A. M., Nehvi, F. A., Wani, S. A., Qadri, H., Dar, Z. A., and Lone, A. A. 2010. Combining ability studies over environments in Rajmash (Phaseolus vulgaris L.) in Jammu and Kashmir, India. J. Plant Breed. Crop Sci. 2 (11): 333-338.

Kempthorne, O., 1957. An Introduction of Genetics Statistics. The Iowa University Press.

Kunkaew, W., Julsrigival, S., Senthong, C., and Kariadee, D. 2006. Estimation of heterosis and combining ability in azukibean (Vigna angularis) under highland growing conditions in
Thailand. CMU Journal. 5(2): 163-168.

Maass, B. L., Knox, M. R., Venkatesha, S. C., Angessa, T. T., Ramme, S., and Pengelly, B. C. 2010. Lablab purpureus - A Crop Lost for Agrica? Tropical Plant Biology. 3: 123-135.

Mahadevu, P., and Byre Gowda, M. 2005. Genetic improvement of Dolichos bean (Lablab purpureus L.) through the use of exotic and indigenous germplasm. Indian Journal of Plant Genetic Resources. 18: 1-5.

Matzinger, D. F., Sprague, G. F., and Cockerham, C. C. 1959. Diallel crosses ofmaize in experiments repeated over locations and years. Agronomy Journal. 51: 346-350.

Mugwira, L. M., and Haque, I. 1993. Screening forage and browse legumes germplasm to nutrient stress: II. Tolerance of Lablab purpureus L. to acidity and low phosphorus in two acid soils, Journal of Plant Nutrition. 16: 3750.

Murphy, A. M., and Colucci, P. E. 1999. A tropical forage solution to poor quality ruminant diets: A review of Lablab purpureus. Livestock Research \& Rural Development. 11: 96-113.

NRC (National Research Council). 2006. Lablab. In: Lost Crops of Africa, Vol II: vegetables. NRC, Washington DC, USA, Pp. 190-205.

Ram, D., and Rajput, C. B. S. 1999. Line x Tester analysis for green pod yieldand its components in French bean (Phaseolus vulgaris L.). Horticulture Journal. 14(2): 155-161.

Rawlings, J. O., and Cockerham, C. C. 1962. Analysis of double cross hybrid populations. Biometrics. 18: 229-244.

Saleem, S. A. 2009. Heterosis and combining ability in a diallel cross of eight faba bean (Vicia faba L.) genotypes. Asian Journal of Crop Science. 1(2):66-76.

Shivashankar, G., and Kulkarni, R. S. 1989. 
Field bean (Dolichos lablab Linn. var. lignosus Prain). Indian Horticulture. 34: 24-27.

Singh, S. P., and Singh, H. N. 1981. Gene system involved and their implication in breeding of lablab bean (Dolichos lablab L). Zeitschirft fun pjflanzenzuchtung. 87: 240-247.

Singh, S. P., Singh, H. N., and Srivastava, J. P. 1980. Combining ability in lablab bean (Lablab purpureus L. Sweet). Indian Agriculture. 30: 147-152.

Singh, S. P., Singh, H. N., and Srivastava, J. P. 1986. Genetic studies of flowers and pods/raceme in hyacinth bean (Dolichos lablab L). Farm Science Journal. 1: 8588.

Sofi, P., Rather, A. G., and Wani, S. A. 2006. Combining ability and gene action studies over environments in field pea (Pisum sativum L.). Pakistan Journal of Biological Sciences. 9(14): 2689-2692.

Stadler, L.J. 1939. Some observations on gene variability and spontaneous mutation. Spragg Memorial Lectures Michigan College. Pp. 1-15.

\section{How to cite this article:}

Keerthi, C.M., S. Ramesh, M. Byregowda and Mohan Rao, A. 2018. Hybrid Specific Combining Ability in Relation to Parental General Combining Ability in Dolichos Bean (Lablab purpureus L.). Int.J.Curr.Microbiol.App.Sci. 7(01): 1442-1450.

doi: https://doi.org/10.20546/ijcmas.2018.701.175 